\title{
Pathogenesis of Lethal Shock After Intravenous Staphylococcal Enterotoxin B in Monkeys
}

\author{
L. F. HODOVAL, ${ }^{1}$ E. L. MORRIS, ${ }^{2}$ G. J. CRAWLEY, ${ }^{3}$ AND W. R. BEISEL \\ U.S. Army Medical Unit, Fort Detrick, Frederick, Maryland 21701
}

Received for publication 10 July 1967

\begin{abstract}
The pathogenesis of shock in the rhesus monkey given intravenous staphylococcal enterotoxin B (SEB) is not understood. Several cardiovascular changes produced by a highly purified preparation of SEB were studied after administration of doses ranging from 50 to $1,000 \mu \mathrm{g} / \mathrm{kg}$. Irreversible arterial hypotensicn was found consistently at the higher doses. Arterial blood pressure and cardiac output declined substantially as shock developed. Total peripheral vascular resistance did not rise at any time, but showed a significant fall during the late stages of shock. Portal and central venous pressures remained essentially unchanged. Venous $\mathrm{O}_{2}$ content and $\mathrm{pO}_{2}$ declined gradually throughout the period of toxemia, but arterial $\mathrm{O}_{2}$ content remained constant until just prior to death, when a slight fall was noted in some monkeys. These changes were consistent with a pooling of blood in the peripheral vascular beds and seemed to resemble cardiovascular responses reported to occur in monkeys during shock due to bacterial endotoxin. Epinephrine, administered in the late stages of shock, caused arterial pressure to increase almost immediately and cardiac output to return to normal about 1 min later. Although life could occasionally be prolonged for several hours by continuous or intermittent epinephrine infusions, this therapy never succeeded in reversing the lethal effects of high doses of SEB.
\end{abstract}

Staphylococcal enterotoxin type B (SEB), an exotoxin long incriminated as a causative substance of certain outbreaks of food poisoning, has been reported to cause lethal shock when injected intravenously into monkeys $(5,7,11)$. Although the relationships between specific toxins and the clinical findings during a generalized staphylococcal infection have not been determined, the possibility exists that SEB, even though produced only in minute quantities, might play a role in the pathogenesis of arterial hypotension during staphylococcal sepsis. Crude staphylococcal toxin, when administered to experimental animals, has been reported to produce shock and an impairment of renal function similar to that observed in patients suffering from staphylococcal septicemia (13).

Previous reports from our laboratory have described several alterations in body functions

1 Present address: Department of Veterinary Physiology, University of Missouri, Columbia, Mo. 65201.

2 Present address: Large Animal Clinics, School of Veterinary Medicine, Texas A\&M University, College Station, Tex.

${ }^{3}$ Present address: Waukesha Veterinary Medical Association, 145 Lake Street, Mukwonago, Wis. 53149. which follow a lethal intravenous challenge of purified SEB. These included the clinical syndrome which terminated in shock $(5,7,11)$, multiple changes in clinical chemistries (4), a hypotension-associated fall in renal function (10), and terminal depression of electroencephalographic patterns (7). Other workers have reported on the pyrogenic and emetic effects $(2,3,14)$.

Information is not currently available concerning details of the cardiovascular response which follows the intravenous (iv) administration of highly purified SEB. Therefore, arterial pressure, central venous pressure, portal pressure, cardiac output, carotid artery flow, and blood oyxgenation were studied in an attempt to delineate the pathophysiology which leads to terminal shock.

\section{Materials AND Methods}

Healthy rhesus monkeys, Macaca mulatta, which weighed 2.5 to $3.5 \mathrm{~kg}$ each and were believed to be free of any previous exposure to SEB, were used. All monkeys were placed in metabolic chairs for 5 to 7 days prior to the start of studies. Food and water were available ad libitum. An indwelling cannula was placed in the saphenous vien under local anesthesia and extended into the posterior vena cava to facilitate 
iv injections and blood sampling. Sodium pentothal was used for general anesthesia. Cannulae and tubing between the test animal and the recording devices contained heparinized saline (20 units $/ \mathrm{ml})$. Pressures were recorded with either a mercury manometer or a strain gauge pressure transducer amplification system (Sanborn Co., Waltham, Mass.). Animals were challenged iv with the same purified preparation of SEB (12) used in other studies performed in this laboratory $(4,5,7,10,11)$. Control animals were studied in a similar manner but received diluent without toxin.

Arterial pressure. Arterial pressures were recorded in 12 monkeys challenged with intermediate (200 to $500 \mu \mathrm{g})$ and high $(1,000 \mu \mathrm{g})$ doses of purified toxin (per kilogram of body weight), while other parameters, such as cardiac output, portal pressures, or carotid artery flow, were being studied. Pressures were recorded from cannulae inserted into the femoral artery the day prior to study and threaded to approximately the descending arch of the aorta. Arterial pressure was also recorded from a carotid artery in 28 additional monkeys which were given lower doses of SEB ( 50 to $200 \mu \mathrm{g} / \mathrm{kg}$ of body weight).

Portal pressure. Portal pressure was measured in three animals challenged with 200,500 , and 1,000 $\mu \mathrm{g}$ of toxin $/ \mathrm{kg}$, respectively. During a laparotomy at least $24 \mathrm{hr}$ prior to challenge, a cannula was threaded from a mesenteric vein into the portal vein. The cannula was exteriorized through the incision for monitoring pressure. The monkeys were allowed to recover from anesthesia and were placed in metabolic chairs; base-line control pressure readings were obtained for several hours prior to the administration of SEB.

Central venous pressure. Venous pressure was recorded in animals after they had received SEB doses that ranged from 50 to $1,000 \mu \mathrm{g} / \mathrm{kg}$. The recordings were obtained via a saphenous vein cannula threaded through the posterior vena cava to approximately the level of the diaphragm.

Cardiac output and carotid artery flow. To study changes in cardiac output, the rate of blood flow in the ascending aortic arch was monitored by a sine wave Statham (R) M-4001 electromagnetic flow meter; the electrical output was continuously recorded by a multichannel Sanborn $550 \mathrm{M}(\mathrm{R})$ polygraph. A surgical approach to the ascending aorta was made through the fourth left intercostal space. After the ascending aorta was freed by blunt dissection, a flow probe, 7 to $9 \mathrm{~mm}$ in diameter, was positioned immediately above the origins of the coronary arteries. The probe leads were exteriorized through the incision.

These animals were allowed 5 to 7 days to recuperate before SEB challenge with $1,000 \mu \mathrm{g} / \mathrm{kg}$. Prechallenge values were obtained and end-diastolic flow was used for determining zero flow. From the values for cardiac output and arterial blood pressure, total peripheral vascular resistance was calculated by the standard formula:

\section{PR $=$ Mean arterial pressure $(\mathrm{mm}$ $\mathrm{Hg}$ )/blood flow in $\mathrm{ml} / \mathrm{sec}$.}

In other animals, the left carotid artery was exposed and a 2-mm electromagnetic flow probe was posi- tioned around the artery; animals were challenged approximately $2 \mathrm{hr}$ later while still under general anesthesia. Zero flow was determined several times during the experiment by occluding the artery with a silk thread.

The flow probes were calibrated after each experiment. Rubber tubing was stretched over each end of the probe, and saline was allowed to flow by gravity directly through the probe in the absence of a vessel. This method was used because it has been reported that neither the vessel wall thickness nor the absence of a vessel affects the recorded flow rate (1). Since preliminary studies with the equipment to be employed confirmed these observations, the technique described above was adopted for routine calibration.

Blood $\mathrm{pO}_{2}$. Because cyanosis develops in conjunction with shock during type $B$ toxemia and because fluids accumulate in interstitial spaces of the lungs, a study of blood $\mathrm{pO}_{2}$ was initiated to determine whether the toxin produced an interference with oxygenation of blood during its circulation through the pulmonary vessels. Oxygen pressure of venous and arterial blood was determined either by the manometric method (15) or with a PHM27 radiometer (R) and PHA927 gas monitor (The London Co., Copenhagen, Denmark). Venous blood samples were obtained anaerobically from the posterior vena cava and arterial blood samples from the descending arch of the aorta. At least two blood samples were obtained prior to challenge, to measure base-line values before each study.

Response to epinephrine. Epinephrine was administered iv to seven monkeys in the late stages of toxemia to determine its effect on cardiac output, carotid artery flow, and arterial blood pressure. Epinephrine $(1 \mathrm{ml})$ was injected $(1: 1,000)$ when the arterial blood pressure declined to 50 to $60 \mathrm{~mm} \mathrm{Hg}$. Either a second injection of epinephrine or an iv infusion $(1: 50,000)$ followed the initial dose. The infusion was administered at a rate to maintain the mean arterial blood pressure above $80 \mathrm{~mm} \mathrm{Hg}$ until the animal became refractory to the epinephrine.

\section{RESULTS}

Irreversible arterial hypotension was a consistent finding at all lethal doses of toxin. Because of the complexities of operative procedures and electronic monitoring methods, it was not feasible to measure all parameters under consideration in a single animal. The varied investigative manipulations also precluded use of these animals to determine exact dose response calculations. However, prior experience indicated that the $\mathbf{L D}_{50}$ for the rhesus monkey fell between 10 and 50 $\mu \mathrm{g} / \mathrm{kg}$ after iv administration. Only rarely did survival occur after doses above $500 \mu \mathrm{g} / \mathrm{kg}$. Since aortic blood pressure changes were found to be the most uniform and consistent manifestation of SEB toxicity, changes in other physiological measurements were related in time and magnitude to alterations in aortic pressure. 
Arterial blood pressure. The prechallenge electronic mean arterial pressures of 40 monkeys averaged $96 \mathrm{~mm} \mathrm{Hg}$ (the $99 \%$ Confidence Interval ranged from 90 to $102 \mathrm{~mm} \mathrm{Hg}$ ). Although the time of death ranged from an hour to as much as $\mathbf{5}$ days after challenge, every nonsurvivor developed progressive arterial hypotension which led to death. Usually, monkeys which were given a higher dose of purified SEB (see Fig. 1) developed severe hypotension more rapidly than those given lower doses. If the mean blood pressure fell below $50 \mathrm{mg} \mathrm{Hg}$ within the first 1 or 2 $\mathrm{hr}$ after challenge, there was little or no spontaneous improvement in the aortic pressure during the remainder of the toxemia. A group of 28 monkeys were challenged with 50 to $200 \mu \mathrm{g} /$ $\mathrm{kg}$; in 18 of these, aortic pressure began to fall within 1 to $4 \mathrm{hr}$ after challenge and gradually declined for 12 to $24 \mathrm{hr}$. After this, five animals had a return of blood pressure to normal before they developed terminal hypotension as late as the 5th postchallenge day. Eight other animals showed no early change in blood pressure and did not exhibit the onset of shock for 3 to 5 days. Two animals that survived the $50 \mu \mathrm{g} / \mathrm{kg}$ dose of toxin failed to exhibit any fall in arterial pressure, whereas three others survived after a period of slight hypotension that disappeared spontaneously. A momentary increase in blood pressure was occasionally noted immediately after administering high doses of the toxin.

The variety of patterns observed are depicted schematically in Fig. 1. Despite the overall variability among dose responses in individual monkeys, higher doses of toxin usually were associated with more rapid onset of shock. Further, any dose of SEB that proved lethal led to the eventual development of irreversible shock.

\section{DOSE RELATED PATTERNS OF SHOCK AFTER S.E.B. (SCHEMATIC)}

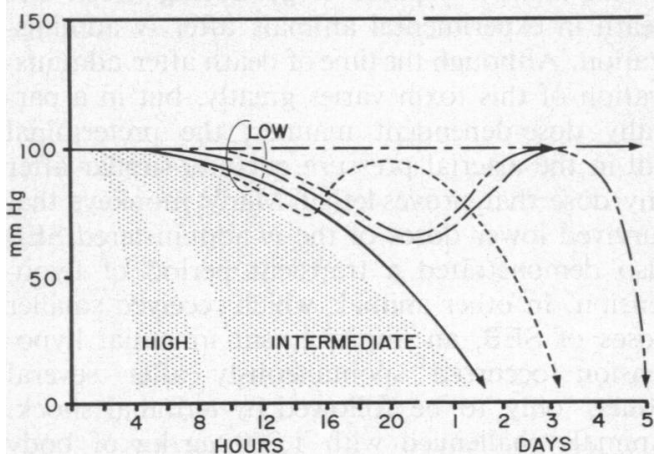

FIG. 1. Alterations of arterial blood pressure after intravenous SEB administration to monkeys; depicted in schematic fashion.
Portal venous pressure. Portal pressures were recorded in three monkeys whose prechallenge values were found to range from 4.5 to $10.0 \mathrm{~mm}$ $\mathrm{Hg}$. They were challenged, respectively, with 200 , 500 , and $1,000 \mu \mathrm{g}$ of SEB $/ \mathrm{kg}$. The monkey which received the lowest dose survived the challenge. In none of these monkeys was any appreciable alteration in portal pressure noted. This finding is similar to that reported after endotoxin administration to monkeys (8), but is quite different from the large increase in portal pressure which resulted from endotoxin injection in dogs $(9 ; \mathbf{L}$. B. Hinshaw et al., Federation Proc., p. 71, 1958).

Venous pressure. Pressures were monitored in the posterior vena cava of 13 monkeys. Challenge doses ranged from 50 to $1,000 \mu \mathrm{g} / \mathrm{kg}$. There was no significant change in central venous pressure of any animal after toxin administration.

Cardiac output and peripheral resistance. Prechallenge cardiac output in nine monkeys averaged $195 \mathrm{ml}$ per min per $\mathrm{kg}$ of body weight. These values showed no detectable change early after the administration of SEB. Cardiac output then decreased gradually during the early stages of the toxemia, and exhibited a fall roughly proportionate to that of arterial blood pressure. As the monkeys progressed into severe shock, arterial blood pressure fell more rapidly than did cardiac output. There were only minor fluctuations in the peripheral resistance of five monkeys until the late stage of toxemia, at which time there was a significant decrease (Table 1).

Carotid flow. Carotid flow was studied in five anesthetized animals. Three monkeys showed an initial brief increase in carotid artery flow immediately after challenge. Carotid flow then decreased in conjunction with developing hypotension, as did cardiac output. Control monkeys which were sham-challenged with the buffer solution used to reconstitute the lyophilized toxin did not show any detectable alteration in the carotid flow.

Epinephrine. During severe SEB shock, epinephrine was capable of reversing hypotension temporarily and of creating a transitory hypertension, as shown in Fig. 2. Such a response pattern was produced several times in four unanesthetized and in two anesthetized monkeys (a seventh monkey died immediately after administration of epinephrine). Cardiac output or carotid flow also returned temporarily to normal after a brief delay. A continuous infusion of epinephrine in saline maintained blood pressure, cardiac output, and carotid flow at slightly below prechallenge values for several hours after the anticipated time of death. After epinephrine was administered, the animals had a marked but 
transient reversal of clinical toxemia; their state of severe depression changed to virtually a normal behavior. Although epinephrine did not prevent toxemic death, it did sustain life for several hours.

Blood pO $\mathrm{O}_{2}$. Partial pressure $\left(\mathrm{pO}_{2}\right)$ of arterial and venous blood from two monkeys was measured with a radiometer. Arterial $\mathrm{pO}_{2}$ did not decrease, although simultaneous central venous $\mathrm{pO}_{2}$ declined gradually but progressively (Fig. 3).

A Van Slyke apparatus was used to determine arterial $\mathrm{O}_{2}$ content in 10 additional animals and central verous $\mathrm{O}_{2}$ content in 3 others. Results similar to the $\mathrm{pO}_{2}$ studies were obtained (Fig. 3); the $\mathrm{O}_{2}$ content of venous blood gradually declined throughout the period of toxemia, whereas the

TABLE 1. Total peripheral vascular resistance in monkeys challenged with 1,000 $\mathrm{\mu g}$ of $S E B$ per $k g$

\begin{tabular}{c|c|c|c|c}
\hline \multirow{2}{*}{ Monkey } & \multicolumn{3}{|c}{ Vascular resistance (mm Hg per ml per sec) } \\
\cline { 2 - 5 } & Control & $\begin{array}{c}30 \text { Min after } \\
\text { toxin }\end{array}$ & $\begin{array}{c}\text { Early } \\
\text { shock }^{a}\end{array}$ & Late shock $^{b}$ \\
\cline { 2 - 5 } & 12.4 & 11.3 & 12.3 & 8.4 \\
A & 11.6 & 12.0 & 12.8 & 7.1 \\
B & 13.2 & 12.7 & 12.2 & 10.4 \\
C & 10.9 & 9.9 & 10.6 & 6.1 \\
D & 12.9 & 12.6 & 11.2 & 6.2 \\
E & 12.2 & 11.7 & 11.8 & 7.6 \\
Mean & 12.7 &
\end{tabular}

a Early shock values were determined when arterial pressure had declined $20 \mathrm{~mm} \mathrm{Hg}$ from control period values.

${ }^{b}$ Late shock resistance was calculated $30 \mathrm{~min}$ prior to death and showed a significant $(P<0.01)$ decline when compared to control values by means of a paired $t$ test.

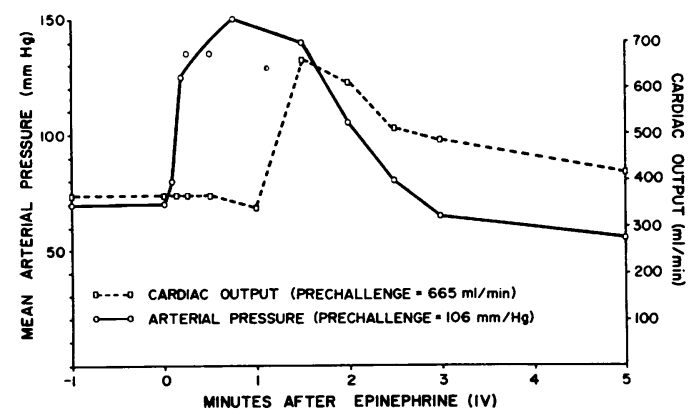

Fig. 2. Transient improvement in cardiac output and arterial pressure after intravenous epinephrine administration during SEB shock. Although arterial pressure rose almost immediately, the increase in cardiac output lagged until approximately 1 min after epinephrine. This response was reproduced several times in individual monkeys.

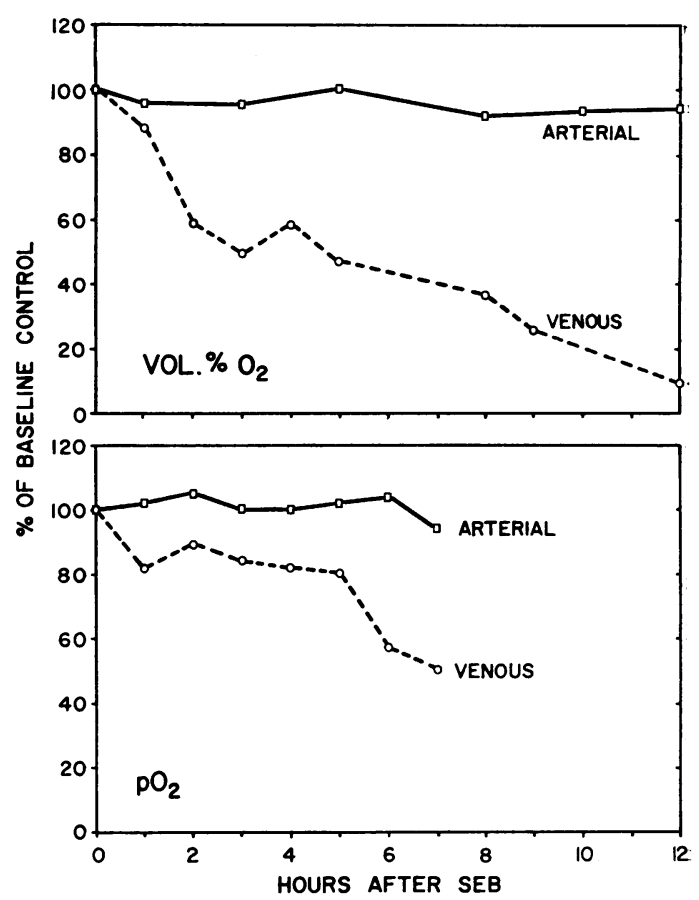

FIG. 3. Blood oxygen content associated with SEB toxemia. Changes from control in the average volume percentage of $\mathrm{O}_{2}$ in arterial and venous blood are shown for 10 and 3 animals, respectively. Arterial $\mathrm{O}_{2}$ content remained normal, whereas venous $\mathrm{O}_{2}$ content decreased. Below are shown similar changes from control in arterial and venous blood $\mathrm{pO}_{2}$ of two monkeys.

arterial $\mathrm{O}_{2}$ concentration did not decrease until immediately before death.

\section{Discuss!on}

Purified SEB initiates the classic symptoms of food poisoning after ingestion; in addition, this potent toxin is capable of producing shock and death in experimental animals after iv administration. Although the time of death after administration of this toxin varies greatly, but in a partially dose-dependent manner, the preterminal fall in the arterial pressure curve is similar after any dose that proves lethal. Many monkeys that survived lower doses of the iv-administered SEB also demonstrated a transient period of hypotension. In other animals which received smaller doses of SEB, an improvement in initial hypotension occurred spontaneously after several hours, only to be followed by terminal shock. Animals challenged with $1,000 \mu \mathrm{g} / \mathrm{kg}$ of body weight demonstrated little, if any, spontaneous reversal of the developing hypotension. Usually, a monophasic pressure curve was recorded at the higher doses. 
The decrease in cardiac output appeared to lag after decreasing arterial pressure. At no time did a spontaneous increase in peripheral resistance become evident. This pattern was similar to that reported for primates given endotoxin, in which a decrease in venous return was implicated as the primary cause of the development of irreversible hypotension (6). Calculations that showed a decrease in peripheral resistance as severe hypotension developed gave evidence for peripheral circulatory failure. The observation that neither portal nor central venous pressure increased during the development of cyanosis and shock indicated that decreased venous return was not due to heart failure or to an impaired egress of portal vein blood from the splanchnic bed. This evidence suggested that peripheral vascular pooling may be a major factor in the developing hypotension as a result of the iv administration of SEB. It cannot be inferred from the data, however, that this purified toxin acts directly on peripheral vascular sites or on endothelial cells; shock could be a secondary manifestation of a toxic action at some other primary location, or one mediated by an agent such as histamine.

The observation that large doses of epinephrine caused an immediate rise in a hypotensive arterial pressure followed by a transitory improvement in cardiac output and carotid flow gave additional indirect evidence that favored peripheral vascular pooling rather than primary cardiac or central nervous system toxicity. Also, it had been observed (7) that abnormal electroencephalographic patterns which developed during SEB toxemia could be reversed by increasing the arterial blood pressure with epinephrine. Such a reversibility suggested that this purified toxin had not directly impaired central nervous system activity but that the electrical alterations were secondary to decreased cerebral blood flow (7). It remains a possibility that the toxin may have caused effects in brain-stem centers that could not be detected by surface electrodes.

The development of cyanosis as a result of interference in gaseous exchange in the lungs was ruled out by the consistent finding that normal arterial blood oxygenation was maintained until the agonal state. This suggested that the cyanotic appearance was the result of red cell $\mathrm{O}_{2}$ loss associated with stagnation of flow through peripheral vascular beds. This concept is substantiated by the progressive decrease in venous $\mathrm{O}_{2}$ content and $\mathrm{pO}_{2}$ during the toxemia.

The pathogenesis of shock in the rhesus monkey given iv SEB is not understood. However, the data obtained in these studies are consistent with the concept that a pooling of blood in peripheral vascular beds is a consistent contributing factor.
Further, changes in the cardiovascular system and in blood $\mathrm{O}_{2}$ content after the administration of this purified protein toxin seemed to resemble closely the changes reported in monkeys after bacterial lipopolysaccharide administration (6). It remains to be determined whether these two bacterial toxins of dissimilar molecular structure evoke a hypotensive response through a similar mechanism. Such a concept implies that each toxin might initiate or trigger an indentical pattern of secondary responses. In this regard, the effects of both SEB and bacterial endotoxin appeared to show a similar, secondary effect on electroencephalographic patterns in the monkey (7). The available evidence does not permit more than speculation concerning the primary site of action of SEB. Although this toxin has been reported to localize in large amounts in the kidney $(10)$, liver $(5,10)$, and lung $(5)$, there is evidence to suggest that no early functional change occurs in any of these organs. Similarly, no primary effect of this toxin seems to occur in the central nervous system, heart, or peripheral arterioles, since their impaired function is rapidly, although transiently corrected by epinephrine administration. Despite the production of vomiting and diarrhea in monkeys after oral SEB, shock or lethality was not observed. This observation reduces the possibility that the gastrointestinal tract might serve as the site of hypotensive effects of the toxin. A primary toxicity involving vascular endothelial permeability has yet to receive positive experimental support, but remains an attractive possibility.

\section{Literature Cited}

1. BeCK, R., J. A. Morris, AND N. S. Assali. 1965. Calibration characteristics of the pulsed-field electromagnetic flowmeter. Am. J. Med. Electron. 4:87-91.

2. Clark, W. G., and H. Borison. 1963. Pyrogenic effect of purified staphylococcal enterotoxin. J. Pharmacol. Exptl. Therap. 142:237-241.

3. Clark, W. G., and A. Cantu. 1966. Pyrogenic effect of staphylococcal enterotoxin B (SEB) in cats. I. Reduction by antiserum (AS). II. Release of endogenous pyrogen. Federation Proc. 25:433.

4. Crawley, C. J., J. N. Black, I. Gray, and J. W. BlANCHARD. 1966. Clinical chemistry of staphylococcal enterotoxin poisoning in monkeys. Appl. Microbiol. 14:445-450.

5. Crawley, G. J., I. Gray, W. A. LeBlang, and J. W. BlanchaRD. 1966. Blood binding, distribution and excretion of staphylococcal enterotoxin in monkeys. J. Infect. Diseases 116:48-56.

6. Hinshaw, L. B., T. E. Emerson, JR., AND D. A REINS. 1966. Cardiovascular response of the primate in endotoxin shock. Am. J. Physiol. 210:335-340. 
7. Hodoval, L. F., E. L. Morris, D. D. Elsberry, AND W. R. BEISEL. 1967. Effect of staphylococcal enterotoxin B on the electroencephalogram of monkeys. Appl. Microbiol. 15:403-406.

8. Kuida, H., R. P. Glibert, L. B. Hinshaw, J. G. Brunson, AND M. B. Visscher. 1961. Species differences in effect of gram-negative endotoxin on circulation. Am. J. Physiol. 200:11971202.

9. MacLean, L. D., AND M. H. Weil. 1956. Hypotension (shock) in dogs produced by Escherichia coli Endotoxin. Circulation Res. 4:546-556.

10. MorRIs, E. L., L. F. Hodoval, AND W. R. BeIsel. 1967. The unusual role of the kidney during intoxication of monkeys by intravenous staphylococcal enterotoxin B. J. Infect. Diseases 117: 273-284.

11. Rapoport, M. I., L. F. Hodoval, E. W. Grogan,
V. MCGANN, AND W. R. BeISEL. 1966. The influence of specific antibody on the disappearance of staphylococcal enterotoxin B from blood. J. Clin. Invest. 45:1365-1372.

12. Schantz, E. J., W. G. Roessler, J. Wagman, L. SPERO, D. A. DUNNERY, AND M. S. BERGDOlL. 1965. Purification of staphylococcal enterotoxin B. Biochemistry 4:1011-1016.

13. SKInNer, D. G., AND M. A. Hayes. 1965. Effect of staphylococcal toxin on renal function: Irreversible shock. Ann. Surg. 162:161-180.

14. Sugiyama, J., K. L. Chow, AND L. R. Dragstedt, II. 1961. Study of emetic receptor sites for staphylococcal enterotoxin in monkeys. Proc. Soc. Exptl. Biol. Med. 108:92-95.

15. Van Slyke, D. D., AND J. M. NeIll. 1924. The determination of gases in blood and other solutions by vacuum extraction and monometric measurement. J. Biol. Chem. 61:523-573. 